\title{
Identifying Clinically Distinct Subgroups of Self-Injurers Among Young Adults: A Latent Class Analysis
}

\author{
E. David Klonsky and Thomas M. Olino \\ Stony Brook University
}

\begin{abstract}
High rates of nonsuicidal self-injury (NSSI; 14\%-17\%) in adolescents and young adults suggest that some self-injurers may exhibit more or different psychiatric problems than others. In the present study, the authors utilized a latent class analysis to identify clinically distinct subgroups of self-injurers. Participants were 205 young adults with a history of 1 or more NSSI behaviors. Latent classes were identified on the basis of method (e.g., cutting vs. biting vs. burning), descriptive features (e.g., self-injuring alone or with others), and functions (i.e., social vs. automatic). The analysis yielded 4 subgroups of self-injurers, which were then compared on measures of depression, anxiety, borderline personality disorder, and suicidality. Almost $80 \%$ of participants belonged to 1 of 2 latent classes characterized by fewer or less severe NSSI behaviors and fewer clinical symptoms. A 3rd class (11\% of participants) performed a variety of NSSI behaviors, endorsed both social and automatic functions, and was characterized by high anxiety. A 4th class (11\% of participants) cut themselves in private, in the service of automatic functions, and was characterized by high suicidality. Clinical and research implications are discussed.
\end{abstract}

Keywords: self-injury, self-mutilation, self-harm, latent class analysis, borderline personality disorder

Nonsuicidal self-injury (NSSI) refers to the intentional and direct injuring of one's own body tissue without suicidal intent. NSSI is listed as a symptom of borderline personality disorder (BPD) by the Diagnostic and Statistical Manual of Mental Disorders (4th ed.; DSM-IV; American Psychiatric Association, 1994) but can also be found in individuals without BPD. Approximately $4 \%$ of the U.S. adult population reports a history of NSSI (Briere \& Gil, 1998; Klonsky, Oltmanns, \& Turkheimer, 2003). Individuals who engage in NSSI are more likely to experience psychiatric problems - such as depression, anxiety, and suicidality — as well as features of borderline and other personality disorders (Andover, Pepper, Ryabchenko, Orrico, \& Gibb, 2005; Klonsky et al., 2003; Whitlock, Eckenrode, \& Silverman, 2006). Thus, NSSI often requires aggressive treatment.

In comparison with adult populations, NSSI appears to be more common in adolescents and young adults. Approximately $14 \%$ of adolescents (Ross \& Heath, 2002) and $17 \%$ of young adults (Whitlock et al., 2006) report a history of one or more NSSI behaviors. These high rates suggest that NSSI does not have the same clinical implications in all cases. Indeed, a study of adolescent inpatients found considerable diagnostic heterogeneity, including that $12 \%$ did not meet criteria for a mental disorder (Nock, Joiner, Gordon, Lloyd-Richardson, \& Prinstein, 2006). It would be

E. David Klonsky and Thomas M. Olino, Department of Psychology, Stony Brook University.

This work was supported in part by a grant from the American Foundation for Suicide Prevention and by the Office of the Vice President of Research at Stony Brook University.

Correspondence concerning this article should be addressed to E. David Klonsky, Department of Psychology, Stony Brook University, Stony Brook, NY 11794-2500. E-mail: E.David.Klonsky@stonybrook.edu useful, then, to investigate large, nonclinical samples of adolescent and young adults and to clarify how many and which self-injurers are likely to have severe psychopathology and require aggressive treatment.

To help explain psychiatric heterogeneity in NSSI, a few researchers have related different manifestations of NSSI to different psychiatric presentations. Andover et al. (2005) found that psychiatric symptoms differed by method of NSSI. Specifically, young adults who utilized skin cutting were found to report more symptoms of anxiety than those who performed other forms of NSSI. Nock and Prinstein (2005) examined the functions of NSSI in relation to clinical presentation. Adolescents endorsing automatic functions (e.g., to stop bad feelings, to feel relaxed) were more likely to have made a recent suicide attempt, feel hopeless, and endorse symptoms of posttraumatic stress disorder. Contextual variables may also be relevant for determining clinical implications of NSSI. For example, Nock et al. (2006) found that utilizing a greater variety of NSSI methods and experiencing less pain during NSSI were both associated with a history of attempted suicide.

On the basis of the studies described, we hypothesized that clinically distinct subgroups of self-injurers could be identified on the basis of NSSI method, function, and contextual features. In the present study, we applied a latent class analysis (LCA) to test this hypothesis. LCA is a method of classifying individuals from a heterogeneous population into smaller, relatively homogenous unobserved subgroups (B. Muthén \& Muthén, 2000). Detailed information on the phenomenology and functions of NSSI was obtained from a large sample of young adults reporting NSSI. These data were entered into an LCA, and the resultant classes were compared on key clinical variables, including depression, anxiety, BPD symptoms, and suicidal behavior. 


\section{Method}

\section{Participants and Procedure}

Participants were 205 college students who endorsed at least one NSSI behavior on a mass screening administered to 815 students in introductory psychology courses. That approximately 1 in 4 participants endorsed NSSI is comparable with previous research on college students (Whitlock et al., 2006). Other measures included in this study were also completed at this time. All participants received course credit, provided informed consent, and had the option of completing an alternative assignment for equivalent credit. The solicitation of participants did not mention NSSI or psychopathology; thus, systematic differences on key study variables between participants and potential participants opting not to complete the mass testing are unlikely. A comparison of study participants to 115 potential participants who skipped the mass screening yielded no significant differences in rates or frequencies of NSSI behaviors.

\section{Measures}

Depression and anxiety. The short version of the Depression Anxiety Stress Scales (DASS-21; Henry \& Crawford, 2005) is a self-report instrument including two 7-item scales that measure depression and anxiety. Each item is rated on a 4-point severity scale. The DASS-21 has excellent psychometric properties (Henry \& Crawford, 2005).

$B P D$. The McLean Screening Instrument for Borderline Personality Disorder (MSI-BPD; Zanarini et al., 2003) is a 10-item self-report measure of the $D S M-I V$ BPD criteria. When compared with a validated structured interview, sensitivity and specificity were both above .90 in a sample of young adults (Zanarini et al., 2003).

Suicidality. The Youth Risk Behaviors Survey (YRBS; Kann, 2001) is administered by the U.S. Center for Disease Control to assess health-risk behaviors, including suicidal behaviors. The YRBS items assessing suicidal thoughts and behavior were utilized in the present study. A history of suicidal ideation was measured by the following item: "Have you ever seriously thought about killing yourself?" A history of attempted suicide was measured by the following item: "Have you ever tried to kill yourself?" Medical severity of past attempts was measured by the following item: "If you have tried to kill yourself, did any attempt result in an injury, poisoning, or overdose that had to be treated by a doctor or nurse?" Participants could answer "yes" or "no" to each question. YRBS suicide questions have been found to have good reliability (Brenner et al., 2002).

History of NSSI. A questionnaire assessed lifetime frequency of 12 different NSSI behaviors performed "intentionally (i.e., on purpose) and without suicidal intent" (i.e., banging/hitting self, biting, burning, carving, cutting, wound picking, needle sticking, pinching, hair pulling, rubbing skin against rough surfaces, severe scratching, and swallowing chemicals). In addition, the questionnaire assessed descriptive features, including age of onset, experience of physical pain, whether self-injury takes place alone or in the presence of others, and time from the urge to self-injure until the NSSI act.

We summarize here this questionnaire's psychometric properties. Reliability and validity were examined in a sample of 761 college students. Internal consistency of the 12 NSSI behaviors was excellent $(\alpha=.84)$. Item-total correlations for the behaviors ranged from .22 (swallowing chemicals) to .60 (banging/hitting self), with a median of .52 . One-to-four week test-retest reliability was examined in a subsample of 59 college students. Test-retest reliability of the omnibus NSSI scale was .85. Spearman correlations between Time 1 and Time 2 reports of lifetime frequency of NSSI behaviors ranged from .54 (pinching) to .94 (interfering with wound healing), with a median of .74 indicating good reliability. To examine construct validity, we correlated the total NSSI score with each item on the MSI-BPD, the total MSI-BPD scale omitting the suicide/self-harm item, and lifetime suicide ideation and attempts as measured by items from the YRBS. As predicted, the NSSI total score correlated more highly with the suicide/self-harm item $(r=.45)$ than with any other MSI-BPD item (median $r=$ .21). Also as predicted, the total NSSI score exhibited a moderate correlation with the MSI-BPD scale (the suicide/self-harm item was omitted; $r=.37)$, as well as with suicide ideation $(r=.38)$ and attempted suicide $(r=.28)$. All correlations were statistically significant at an alpha level of .001.

Functions of NSSI. A recent empirical review has noted that the field lacks a comprehensive measure of NSSI functions (Klonsky, 2007). The Inventory of Statements about Self-Injury was developed on the basis of this review and used in the present study. Items equivalent or similar to items used in previous studies were pooled to measure a variety of functions. The functions assessed in the present study were as follows: (a) affect regulation, (b) selfpunishment, (c) anti-dissociation, (d) anti-suicide, (e) interpersonal influence, (f) peer bonding, (g) sensation seeking, and (h) interpersonal boundaries. These functions are described in Klonsky's (2007) study.

Each function on the Inventory of Statements about Self-Injury is assessed by three items. Participants rate how well items complete the phrase: "When I harm myself, I am ...." Examples of items and the functions they assess are as follows: "calming myself down" (affect regulation), "punishing myself" (self-punishment), "seeking care or help from others" (interpersonal influence), and "fitting in with others" (peer bonding). Participants rate each item on a 3-point scale as very relevant, somewhat relevant, or not relevant. The questionnaire takes approximately $8 \mathrm{~min}$ to complete.

An exploratory factor analysis with promax rotation revealed two superordinate factors that were consistent with previous research (Nock \& Prinstein, 2004). The first factor (eigenvalue = 3.5) represented socially reinforcing functions (interpersonal influence, peer bonding, sensation seeking, and interpersonal boundaries). The second factor (eigenvalue $=1.4$ ) represented automatically reinforcing functions (affect regulation, self-punishment, anti-suicide, and anti-dissociation). These were the only two factors with eigenvalues greater than one. Inspection of the scree plot also indicated that two factors should be retained. The two-factor solution accounted for $61 \%$ of the variance. Additional data on this instrument's psychometric properties are being prepared for publication (Glenn \& Klonsky, 2007).

\section{Data Analysis}

We performed the LCA using Mplus, Version 4.1 (L. K. Muthén \& Muthén, 1998-2006). Model solutions were evaluated on the 
Table 1

Lifetime Frequency of 12 NSSI Behaviors in the Full Sample of Self-Injurers $(n=205)$

\begin{tabular}{lcccc}
\hline \multicolumn{1}{c}{ Type of behavior } & $\begin{array}{c}\text { times } \\
(\%)\end{array}$ & $\begin{array}{c}1 \text { or } 2 \\
\text { times }(\%)\end{array}$ & $\begin{array}{c}3-10 \\
\text { times }(\%)\end{array}$ & $\begin{array}{c}\text { More than } 10 \\
\text { times }(\%)\end{array}$ \\
\hline Banging or hitting self & 39 & 11 & 20 & 30 \\
Hair pulling & 53 & 6 & 19 & 23 \\
Pinching & 58 & 7 & 17 & 18 \\
Cutting & 60 & 12 & 17 & 11 \\
Biting & 61 & 11 & 14 & 13 \\
Wound picking & 65 & 1 & 16 & 20 \\
Severe scratching & 68 & 3 & 8 & 11 \\
Rubbing skin against rough surfaces & 83 & 7 & 5 & 6 \\
Burning & 84 & 4 & 5 & 2 \\
Needle sticking & 87 & 5 & 4 & 2 \\
Carving & 88 & 3 & 4 \\
Swallowing chemicals & 92 & 5 & 2 \\
\hline
\end{tabular}

Note. $\quad$ NSSI $=$ nonsuicidal self-injury.

basis of the Bayesian information criterion (BIC) and entropy (B. Muthén \& Muthén, 2000). On the basis of a recent simulation study, the BIC performed better than other information criteria and likelihood ratio tests in identifying the appropriate number of latent classes (Nylund, Asparouhov, \& Muthén, 2007). Better fitting models have lower BIC values. Entropy is an index for assessing the precision of assigning latent class membership. Higher probability values indicate greater precision of classification.

\section{Results}

\section{Sample Characteristics}

Mean age of the sample was 18.5 years $(S D=1.2)$. Of the participants, $57 \%$ were female. Racial composition of the sample was as follows: $42 \%$ Caucasian, 39\% Asian, 6\% African American, 5\% Hispanic, $1 \%$ Native American, and the remaining participants indicated their race as "other." Frequencies for the different NSSI behaviors are presented in Table 1. Banging or hitting oneself was the most common form of NSSI followed by hair pulling, pinching, cutting, and biting. Of the sample, $63 \%$ had self-injured within the past year.

\section{Extraction of Latent Classes}

Indicators of the LCA were lifetime presence of 12 NSSI behaviors (cutting, biting, burning, carving, pinching, hair pulling, scratching, banging/hitting, wound picking, rubbing skin, needle sticking, and swallowing), descriptive features (absence of pain, whether NSSI occurs exclusively while alone, time from the urge to self-injure until the NSSI act), and two superordinate functions of NSSI (social and automatic reinforcement).

LCAs were conducted that specified 2-10 classes. The best fitting model, as indicated by the BIC, was the four-class model. ${ }^{1}$ The entropy value for the four-class model was high (.912), which suggests that there was great precision in assigning individual cases into their appropriate class. As shown in Table 2, this model included the following: (a) a class of individuals with low probabilities of NSSI behaviors, except a moderate-high probability of lifetime banging or hitting oneself, and relatively low levels of socially reinforcing and automatically reinforcing functions; (b) a class of individuals with high probabilities of lifetime biting, pinching, hair pulling, and banging or hitting oneself; a moderatehigh probability of lifetime of scratching, wound picking, and rubbing skin; and relatively low levels of automatically and socially reinforcing functions; (c) a class of individuals with moderate-high probabilities of numerous NSSI behaviorsincluding banging or hitting oneself, biting, cutting, hair pulling, pinching, and scratching - and high levels of both socially reinforcing and automatically reinforcing functions; and (d) a class of individuals with a high probability of cutting, with a moderatehigh probability of wound picking and needle sticking, with high levels of automatic but not socially reinforcing functions, and who almost exclusively self-injure when alone. Without exception, as determined by a series of analyses of variance (ANOVAs), ${ }^{2}$ latent classes that had the highest probability of engaging in a particular NSSI behavior also engaged in that behavior the most frequently.

\section{Comparison of Classes}

After extracting the four latent classes, individuals were assigned to their most likely class and were compared on a number of clinical measures. We conducted group comparisons using one-way ANOVAs and post hoc least significant difference tests,

\footnotetext{
${ }^{1}$ The four-class solution was selected on the basis of the BIC; however, the bootstrap likelihood ratio test, Lo-Mendell-Rubin likelihood ratio test, and entropy provided conflicting results. The four-class solution was compared on theoretical and empirical grounds to alternative solutions and appeared more parsimonious and defensible. Additionally, in analyses that included large numbers of starting values $(>100)$, we found that the data achieved a global, rather than local, solution. Finally, we note that the model examined posits that all associations between observed variables within the latent classes are due to latent class membership. However, this is a rather rigorous assumption, which is often unrealistic. A more detailed technical account of the model-fitting process is available to readers upon request from E. David Klonsky.

${ }^{2}$ Detailed results of ANOVAs comparing frequencies of each of the 12 NSSI behaviors across the four latent classes are available upon request from E. David Klonsky.
} 
Table 2

Characteristics of NSSI in the Four Latent Classes of Self-Injurers

\begin{tabular}{|c|c|c|c|c|}
\hline Characteristic & $\begin{array}{c}\text { Class 1 } \\
(n=125)\end{array}$ & $\begin{array}{c}\text { Class 2 } \\
(n=35)\end{array}$ & $\begin{array}{c}\text { Class } 3 \\
(n=23)\end{array}$ & $\begin{array}{c}\text { Class } 4 \\
(n=22)\end{array}$ \\
\hline \multicolumn{5}{|l|}{ Method, ${ }^{\mathrm{a}} n(\%)$} \\
\hline Banging/hitting & $70(56.0)$ & $33(94.3)$ & $14(60.9)$ & $8(38.1)$ \\
\hline Hair pulling & $48(38.7)$ & $30(85.7)$ & $12(52.2)$ & $6(28.6)$ \\
\hline Pinching & $41(32.8)$ & $33(94.3)$ & $12(52.2)$ & $0(0.0)$ \\
\hline Cutting & $34(27.2)$ & $16(45.7)$ & $12(52.2)$ & $20(90.9)$ \\
\hline Biting & $34(27.2)$ & $33(94.3)$ & $12(52.2)$ & $0(0.0)$ \\
\hline Wound picking & $31(24.8)$ & $21(60.0)$ & $8(34.8)$ & $12(57.1)$ \\
\hline Scratching ${ }^{\mathrm{b}}$ & $22(17.6)$ & $23(65.7)$ & $12(52.2)$ & $9(42.9)$ \\
\hline Rubbing skin ${ }^{\mathrm{c}}$ & $7(5.6)$ & $18(51.4)$ & $7(30.4)$ & $3(14.3)$ \\
\hline Burning & $8(6.4)$ & $14(40.0)$ & $4(17.4)$ & $7(33.3)$ \\
\hline Needle sticking & $0(0.0)$ & $9(25.7)$ & $6(26.1)$ & $11(52.4)$ \\
\hline Carving & $4(3.2)$ & $8(22.9)$ & $3(13.0)$ & $10(47.6)$ \\
\hline Swallowing $^{\mathrm{d}}$ & $3(2.4)$ & $0(0.0)$ & $6(26.1)$ & $8(38.1)$ \\
\hline \multicolumn{5}{|c|}{ Descriptive features, $n(\%)$} \\
\hline Pain $^{\mathrm{e}}$ & $32(26.7)$ & $6(17.1)$ & $5(21.7)$ & $1(4.5)$ \\
\hline Alone $^{f}$ & $56(47.5)$ & $20(57.1)$ & $11(47.8)$ & $21(95.5)$ \\
\hline Time to act ${ }^{\mathrm{g}}$ & $38(33.9)$ & $10(29.4)$ & $6(27.3)$ & $13(61.9)$ \\
\hline \multicolumn{5}{|l|}{ Functions, $M(S D)$} \\
\hline Social ${ }^{\mathrm{h}}$ & $0.30(.40)$ & $0.39(.41)$ & $3.25(.79)$ & $0.72(.55)$ \\
\hline Automatic $^{\mathrm{h}}$ & $1.39(1.05)$ & $1.89(1.29)$ & $3.39(1.06)$ & $3.06(1.39)$ \\
\hline
\end{tabular}

${ }^{a}$ Indicates the percentage of participants within each class who performed each method of nonsuicidal self-injury (NSSI). ${ }^{\mathrm{b}}$ Indicates severe skin scratching. ${ }^{\mathrm{c}}$ Indicates rubbing skin against rough surfaces. ${ }^{\mathrm{d}}$ Indicates swallowing dangerous chemicals. ${ }^{\mathrm{e}}$ Indicates the percentage of participants who did not experience physical pain during NSSI. ${ }^{\mathrm{f}}$ Indicates the percentage of participants who self-injured only when alone. ${ }^{\mathrm{g}}$ Indicates the percentage of participants who typically waited more than an hour from the urge to self-injure until the act. ${ }^{\mathrm{h}} \mathrm{A}$ score of " 0 " indicates that no items on the scale were endorsed, and a score of " 8 " indicates maximum endorsement of items on a scale.

as well as chi-square tests. Results of these analyses are presented in Table 3.

Significant differences among the classes were observed for the age of onset of NSSI, $F(3,172)=3.77, p<.05$; depressive symptoms, $F(3,204)=5.20, p<.001$; anxiety symptoms, $F(3$, $204)=9.73, p<.001$; and BPD symptoms, $F(3,204)=4.73, p<$
.05. Post hoc comparisons demonstrated that Class 2 had onset of NSSI significantly earlier than Class 1 and Class 4 . The onset of NSSI for Class 3 was also earlier than Class 4. Class 1 had the lowest levels of depressive symptoms, which did not differ from those of Class 2. Levels of depressive symptoms in Class 3 and Class 4 were significantly higher than in Class 1 . Class 3 had

Table 3

Clinical Differences Among the Four Latent Classes of Self-Injurers

\begin{tabular}{|c|c|c|c|c|c|c|c|}
\hline Variable & $\begin{array}{c}\text { Class 1 } \\
(n=125)\end{array}$ & $\begin{array}{c}\text { Class 2 } \\
(n=35)\end{array}$ & $\begin{array}{c}\text { Class } 3 \\
(n=23)\end{array}$ & $\begin{array}{c}\text { Class } 4 \\
(n=22)\end{array}$ & $F$ & $d f \mathrm{~s}$ & $\chi^{2}(3)$ \\
\hline Age of onset ${ }^{\mathrm{a}}$ & & & & & $3.77^{*}$ & 3,172 & \\
\hline$M(S D)$ & $13.13(3.34)_{\mathrm{a}, \mathrm{b}}$ & $11.52(3.14)_{\mathrm{c}}$ & $11.65(3.22)_{\mathrm{a}, \mathrm{c}}$ & $13.81(1.25)_{\mathrm{b}}$ & & & \\
\hline Depression $^{\mathrm{b}}$ & & & & & $5.20^{*}$ & 3,204 & \\
\hline$M(S D)$ & $4.42(4.84)_{a}$ & $5.77(4.93)_{\mathrm{a}, \mathrm{b}}$ & $8.43(5.87)_{b}$ & $7.27(6.26)_{\mathrm{b}}$ & & & \\
\hline Anxiety ${ }^{\mathrm{b}}$ & & & & & $9.73^{* * * *}$ & 3,204 & \\
\hline $\begin{array}{l}M(S D) \\
p^{c}\end{array}$ & $4.23(4.18)_{\mathrm{a}}$ & $4.74(3.16)_{a, b}$ & $9.17(5.29)_{\mathrm{c}}$ & $6.50(4.78)_{\mathrm{b}}$ & & & \\
\hline $\mathrm{BPD}^{\mathrm{c}}$ & & & & & $4.73^{* * *}$ & 3,204 & \\
\hline $\begin{array}{l}M(S D) \\
\text { Suicide, } n(\%)\end{array}$ & $4.33(2.46)_{a}$ & $5.43(2.82)_{\mathrm{b}}$ & $.26(2.86)_{a, b}$ & $6.27(2.47)_{\mathrm{b}}$ & & & \\
\hline Ideation $^{\mathrm{d}}$ & $50(40.0)_{\mathrm{a}}$ & $19(54.3)_{\mathrm{a}, \mathrm{b}}$ & $12(52.2)_{\mathrm{a}, \mathrm{b}}$ & $17(77.3)_{\mathrm{b}}$ & & & $11.47^{* *}$ \\
\hline Attempt ${ }^{\mathrm{e}}$ & $16(12.8)_{\mathrm{a}}$ & $6(17.1)_{a}$ & $6(26.1)_{\mathrm{a}, \mathrm{b}}$ & $10(45.5)_{\mathrm{b}}$ & & & $14.19^{* *}$ \\
\hline Medical $^{\mathrm{f}}$ & $7(5.7)_{\mathrm{a}}$ & $1(2.9)_{\mathrm{a}}$ & $3(13.0)_{\mathrm{a}, \mathrm{b}}$ & $7(31.8)_{\mathrm{b}}$ & & & $17.94^{* * * *}$ \\
\hline
\end{tabular}

Note. For each row, cell values that do not share subscripts are significantly different according to post hoc tests. BPD $=$ borderline personality disorder. ${ }^{a}$ Indicates the age at which nonsuicidal self-injury was first performed. ${ }^{\mathrm{b}}$ As measured by the Depression Anxiety Stress Scales. ${ }^{\mathrm{c}}$ As measured by the McLean Screening Instrument for Borderline Personality Disorder. ${ }^{\mathrm{d}}$ Indicates lifetime suicidal ideation as measured by the following Youth Risk Behaviors Survey (YRBS) item: "Have you ever seriously thought about killing yourself?" e Indicates lifetime attempt status as measured by the following YRBS item: "Have you ever tried to kill yourself?" ' Indicates whether medical treatment was required as measured by the following YRBS item: "If you have tried to kill yourself, did any attempt result in an injury, poisoning, or overdose that had to be treated by a doctor or nurse?" ${ }^{*} p<.05 .{ }^{* * *} p<.01 .^{* * *} p<.001$. 
significantly higher levels of anxiety symptoms than the other classes. Class 4 had significantly higher levels of anxiety than Class 1. Lastly, Class 4 and Class 2 had significantly higher levels of BPD symptoms compared with Class 1 . A chi-square test found gender differences among the classes at a trend level, $\chi^{2}(3, N=$ 204) $=7.36, p=.06$. There was a higher proportion of women in Class $4(82 \%)$ compared with Class 1 (53\%) and Class $3(50 \%)$. Class 2 was composed of $63 \%$ women. Ethnic composition (i.e., proportion of Caucasians) did not differ among the classes, $\chi^{2}(3$, $N=204)=3.73, p=.29$.

In addition, significant differences were found for aspects of lifetime suicidality, including ideation, $\chi^{2}(3, N=205)=11.47$, $p<.01$; attempt, $\chi^{2}(3, N=205)=14.19, p<.01$; and requiring medical attention, $\chi^{2}(3, N=203)=17.94, p<.001$. Class 4 had a significantly greater proportion of individuals with lifetime suicidal ideation compared with Class 1 . Class 4 had a significantly greater proportion of individuals who had attempted suicide compared with Class 1 and Class 2. Class 4 also had a higher proportion of individuals who had required medical attention for a suicide attempt than Class 1 or Class 2. The proportion of individuals who had self-injured in the last 12 months is comparable across latent classes, $F(3,151)=1.87, p=.14$, and thus not driving the pattern of results regarding the clinical variables.

\section{Discussion}

In the present, exploratory study, we sought to identify distinct subgroups of self-injurers on the basis of the method, descriptive features, and function of NSSI in young adults. An LCA identified four subgroups, which differed on key clinical variables. The first two classes, which comprised almost $80 \%$ of self-injurers, exhibited fewer clinical symptoms. This result is consistent with our earlier speculation that some self-injurers have fewer psychiatric symptoms than others. Notably, these two classes contained some individuals who had performed more severe forms of NSSI (e.g., cutting, carving).

The first group, comprising $61 \%$ of participants, performed relatively few NSSI behaviors and displayed the fewest clinical symptoms. Members of this group may be those who experimented with NSSI on a few occasions, as opposed to those who self-injure more chronically in response to psychiatric distress. This class may be regarded an "experimental NSSI" group.

In comparison with the first group, the second group (17\% of the sample) had an earlier onset of NSSI and performed more NSSI behaviors, particularly biting, pinching, and banging/hitting. Therefore, the NSSI in this group appears to represent more than just occasional experimentation. This group also endorsed slightly more BPD symptoms than the first group, although the overall level of clinical symptomatology was relatively low. That the NSSI behaviors most characteristic of this group could be considered less severe as compared with cutting or swallowing chemicals may account for the absence of severe psychopathology. This class may be regarded as a "mild NSSI" group.

The third and fourth groups identified by the LCA comprised $22 \%$ of the sample. Both exhibited increased clinical symptomatology. To the extent that the results generalize, we could extrapolate that approximately one in five young adults who have engaged in NSSI have heightened psychiatric problems requiring more aggressive treatment.
The third group (11\% of the sample) utilized a variety of NSSI methods, such as banging/hitting, biting, cutting, hair pulling, pinching, and scratching. This group also heavily endorsed both social and automatic functions, suggesting that these behaviors were multiply reinforced. Clinically, members of this group had an early onset of NSSI and displayed more symptoms of anxiety than any other group. Thus, this class may be regarded as a "multiple functions/anxious" group. From a treatment perspective, the early age of onset and overdetermined nature of the NSSI suggest that treatment of NSSI could be particularly difficult. To the extent that NSSI serves to alleviate the elevated anxiety observed in this class, helping clients reduce and better cope with anxiety would decrease the need for and occurrence of NSSI.

The fourth group (10\% of the sample) almost exclusively comprised those who cut themselves in private in the service of automatic functions. NSSI also appeared to be less impulsive in this group, as $60 \%$ reported that a typical instance of NSSI would occur more than $1 \mathrm{hr}$ after the urge to self-injure. The typical latency in the first three groups was less than $1 \mathrm{hr}$. In light of the increased latency from urge to act and the heavy endorsement of automatic functions (which largely reflect emotion regulation; Klonsky, 2007; Nock \& Prinstein, 2004), we suggest that members of this group often utilize NSSI as a deliberate, premeditated strategy to regulate negative emotions. As might be expected, individuals in this group exhibited many symptoms of depression, anxiety, and BPD. These individuals were also particularly likely to have attempted suicide and to have required medical treatment for a suicide attempt. Of the individuals in this group, $46 \%$ reported having attempted suicide, and $32 \%$ reported having been treated by a doctor or nurse as a result of injury, poisoning, or overdose associated with a suicide attempt. This class may represent an "automatic functions/suicidal" group. In light of the functions endorsed, treatment for members of this class could include teaching emotion regulation strategies other than NSSI. That these individuals tend to let time elapse between NSSI urges and acts will afford opportunities to implement these strategies. Treatment should also involve close monitoring of suicidal thoughts and the relationship of NSSI to suicidal thoughts and behaviors.

Findings from the present study have important clinical implications. On the one hand, results suggest that NSSI in young adults is not always accompanied by severe psychiatric symptoms. On the other hand, many who have self-injured are in considerable psychiatric distress. Therefore, thorough clinical assessments are necessary for adolescents and young adults who have self-injured, especially if NSSI was the sole reason for treatment. For example, a teacher or counselor who discovers that a student has self-injured might feel compelled to recommend intensive mental health treatment regardless of the NSSI's nature or associated clinical features. The clinician must carefully determine which and whether treatment is the optimal course of action.

In addition, some researchers suggest that NSSI belongs on the same spectrum as attempted suicide (Stanley, Winchel, Molcho, Simeon, \& Stanley, 1992) and that individuals who present to treatment with a history of NSSI should be regarded as carrying significant risk of fatality (Fortune, 2006). Clinicians adhering to these notions may be inclined to hospitalize individuals presenting with NSSI. Results from the present study suggest that great care should be taken to select a treatment matching the clinical profile (e.g., inpatient treatment for severe self-injury with comorbid 
suicidality, outpatient treatment for moderate self-injury accompanied by depression but not suicidal ideation or intent) as well as to avoid prescribing treatment when it is not indicated (e.g., occasional, experimental NSSI in the absence of other clinical symptoms). Of course, even if an initial instance of NSSI does not warrant formal mental health treatment, it is important to ensure that the NSSI does not continue or signal the beginning of a chronic condition. Therefore, if an initial decision is made to not initiate mental health treatment, a follow-up assessment might be useful to ensure problems with NSSI or related psychiatric issues have not persisted or escalated.

In sum, in the present study we utilized LCA as a means of clarifying diagnostic heterogeneity in young adults who have self-injured. Limitations of the study suggest important directions for future research. First, in the present study we utilized a nonclinical, college sample to conduct an initial LCA of self-injury. It is important for future research to seek to replicate findings. It would be particularly instructive if an LCA found a tendency for treatment-seeking self-injurers to resemble the clinical profiles of the third or fourth classes observed in the present study. Such a finding would provide further evidence that these two classes reflect the NSSI profiles most indicative of severe psychopathology and a need for aggressive treatment. Second, in the present study we examined a limited set of clinical variables using selfreport questionnaires. In examining the clinical implications of different latent classes, future researchers should investigate a wider range of potentially relevant variables, including eating disorders, substance disorders, psychotic disorders, and personality disorders other than BPD. When possible, structured diagnostic interviews should be applied to maximize reliability and validity of the diagnostic assessments.

Third, although results from the LCA are suggestive that different subgroups of self-injures may have different prognoses, in the present study we only collected data at a single time point. Some participants may have exhibited few clinical symptoms because they no longer self-injured and had more prominent clinical symptoms when their NSSI was active. About one third of the sample had not self-injured in the past 12 months. Follow-up analyses found that the proportion of individuals who had and had not self-injured in the last 12 months was comparable across the latent classes and, thus, probably not driving the results. Nevertheless, future research should address this limitation. For example, it would be useful for longitudinal studies to compare the trajectories of NSSI and clinical symptoms for different latent classes. Such research could aid in the early identification of self-injurers at greatest risk for developing psychopathology and suicidal behavior, and thereby facilitate prevention and treatment efforts.

\section{References}

American Psychiatric Association. (1994). Diagnostic and statistical manual of mental disorders (4th ed.). Washington, DC: Author.

Andover, M. S., Pepper, C. M., Ryabchenko, K. A., Orrico, E. G., \& Gibb. B. E. (2005). Self-mutilation and symptoms of depression, anxiety, and borderline personality disorder. Suicide and Life-Threatening Behavior, $35,581-591$.
Brenner, N. D., Kann, L., McManus, T., Kinchen, S. A., Sundberg, E. C., \& Ross, J. G. (2002). Reliability of the 1999 Youth Risk Behavior Survey questionnaire. Journal of Adolescent Health, 31, 336-342.

Briere, J., \& Gil, E. (1998). Self-mutilation in clinical and general population samples: Prevalence, correlates, and functions. American Journal of Orthopsychiatry, 68, 609-620.

Fortune, S. A. (2006). An examination of cutting and other methods of DSH among children and adolescents presenting to an outpatient psychiatric clinic in New Zealand. Clinical Child Psychology and Psychiatry, 11, 407-416.

Glenn, C. R., \& Klonsky, E. D. (2007, May). The functions of non-suicidal self-injury: Measurement and structure. Paper presented at the annual meeting of the Association for Psychological Science, Washington, DC.

Henry, J. D., \& Crawford, J. R. (2005). The short-form version of the Depression Anxiety Stress Scales (DASS-21): Construct validity and normative data in a large non-clinical sample. British Journal of Clinical Psychology, 44, 227-239.

Kann, L. (2001). The Youth Risk Behavior Surveillance System: Measuring health-risk behaviors. American Journal of Health Behavior, 25, 272-277.

Klonsky, E. D. (2007). The functions of deliberate self-injury: A review of the evidence. Clinical Psychology Review, 27, 226-239.

Klonsky, E. D., Oltmanns, T. F., \& Turkheimer, E. (2003). Deliberate self-harm in a nonclinical population: Prevalence and psychological correlates. American Journal of Psychiatry, 160, 1501-1508.

Muthén, B., \& Muthén, L. K. (2000). Integrating person-centered and variable centered analyses: Growth mixture modeling with latent trajectory classes. Alcoholism: Clinical and Experimental Research, 24, 882891.

Muthén, L. K., \& Muthén, B. (1998-2006). Mplus user's guide (4th ed.). Los Angeles: Author.

Nock, M. K., Joiner, T. E., Gordon, K. H., Lloyd-Richardson, E., \& Prinstein, M. (2006). Non-suicidal self-injury among adolescents: Diagnostic correlates and relation to suicide attempts. Psychiatry Research, 144, 65-72.

Nock, M. K., \& Prinstein, M. J. (2004). A functional approach to the assessment of self-mutilative behavior. Journal of Consulting and Clinical Psychology, 72, 885-890.

Nock, M. K., \& Prinstein, M. J. (2005). Contextual features and behavioral functions of self-mutilation among adolescents. Journal of Abnormal Psychology, 114, 140-146.

Nylund, K. L., Asparouhov, T., \& Muthén, B. (2007). Deciding on the number of classes in latent class analysis and growth mixture modeling: A Monte Carlo simulation study. Structural Equation Modeling, 14, 535-569.

Ross, S., \& Heath, N. (2002). A study of the frequency of self-mutilation in a community sample of adolescents. Journal of Youth and Adolescence, 31, 67-77.

Stanley, B., Winchel, R., Molcho, A., Simeon, D., \& Stanley, M. (1992). Suicide and the self-harm continuum: Phenomenological and biochemical evidence. International Review of Psychiatry, 4, 149-155.

Whitlock, J., Eckenrode, J., \& Silverman, D. (2006). Self-injurious behaviors in a college population. Pediatrics, 117, 1939-1948.

Zanarini, M. C., Vujanovic, A., Parachini, E. A., Boulanger, J. L., Frankenburg, F. R., \& Hennen, J. (2003). A screening measure for BPD: The Mclean Screening Instrument for Borderline Personality Disorder. Journal of Personality Disorders, 17, 568-573.

Received February 1, 2007

Revision received November 2, 2007

Accepted November 5, 2007 